\title{
Conocimiento de los estudiantes de enfermería sobre las medidas de prevención en infecciones nosocomiales
}

\author{
García-Becerra Ricardo Alejandro*, Castrejón-Reyes Victorina**, Hernández-Castañón Ma. Alejandra**, \\ Garza-González Beatriz**
}

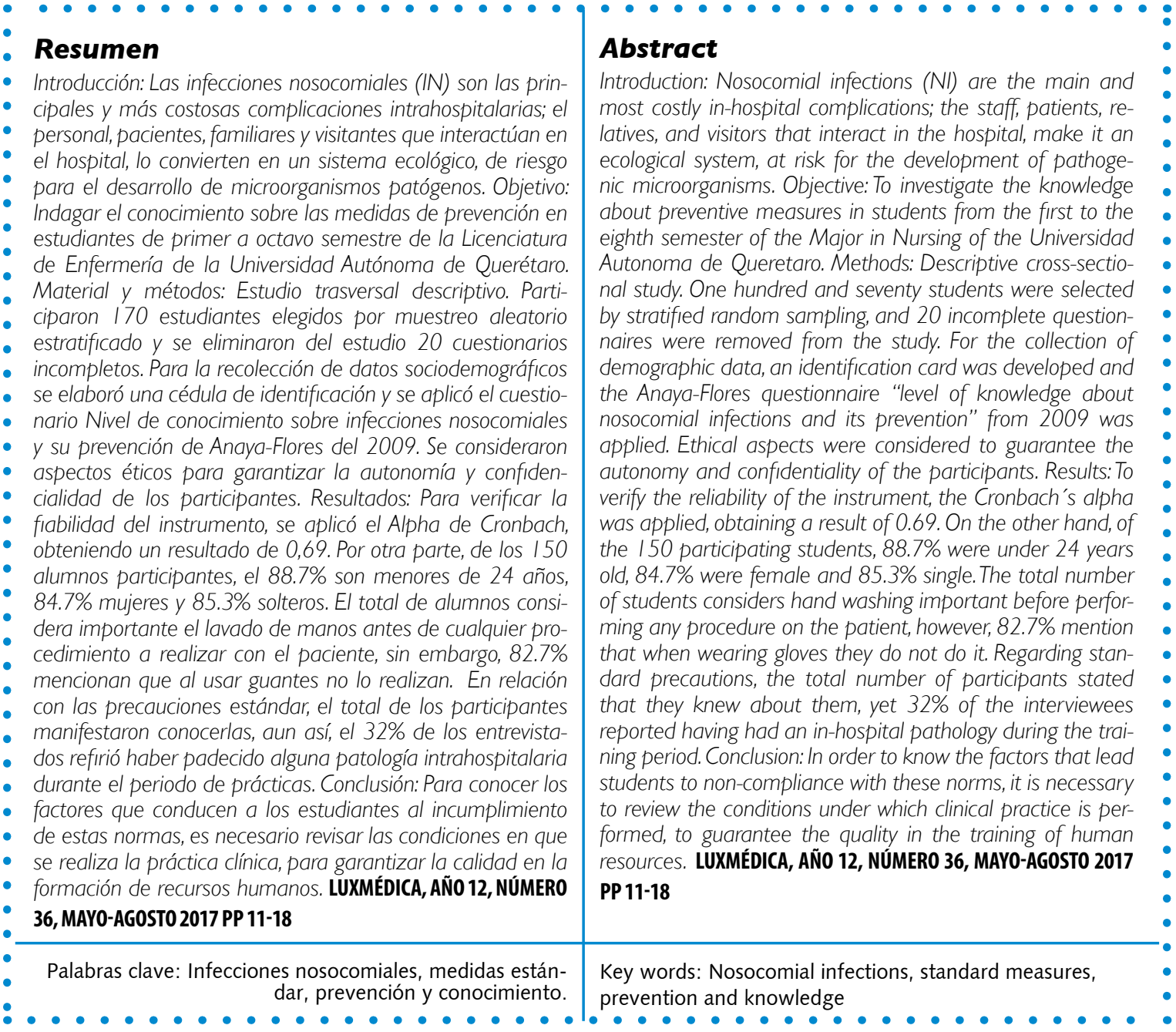

* Pasante de Servicio Social de la Licenciatura de Enfermería de la Facultad de Enfermería. Universidad Autónoma de Querétaro

** Profesoras investigadoras de tiempo completo de la Facultad de Enfermería de la Universidad Autónoma de Querétaro.

Fecha de recibido: 10 de febrero 2017

Fecha de aceptación: 15 de abril 2015

Correspondencia: Ma Alejandra Hernández Castañón. Facultad de Enfermería. Universidad Autónoma de Querétaro. Cerro de las Campanas sin número. Las Campanas Código postal 76010. Santiago de Querétaro, México Teléfono 014421921200 Correo electrónico alehdez983@yahoo.com.mx 


\section{Introducción}

Las infecciones nosocomiales (IN) son asociadas a los cuidados y constituyen un problema relevante de salud pública de trascendencia económica y social, constituyendo un gran desafío para las instituciones de salud y el personal responsable de su atención. ${ }^{1}$ Históricamente las infecciones han acompañado a los hospitales con mayor o menor incidencia, siendo un motivo de preocupación a escala mundial. ${ }^{2}$

Los factores que condicionan las IN son: el agente etiológico, la transmisión y el huésped. En el individuo, la evolución del proceso infeccioso está determinada por la resistencia, el estado nutricional, estrés, edad, sexo, días de internación y patología de base. Mientras que por parte del agente influye la inefectividad y virulencia. ${ }^{3}$ Aunado a lo anterior, las IN se asocian con altas tasas de morbilidad y mortalidad, lo que se traduce no sólo en un incremento en los días de hospitalización y los costos de atención, sino también en un incremento en años de vida ajustados de discapacidad (DALYS) en la población. ${ }^{4}$

Una elevada frecuencia de IN, muestra una calidad deficiente en la prestación de servicios de atención de salud y ocasiona costos evitables. Las IN surgen como consecuencia directa en la mayoría de los casos de la atención en salud, debido a que la medicina invasiva que se practica en la actualidad se asocia con un alto riesgo de infecciones, aunado a que se realizan procedimientos diagnósticos o terapéuticos que por algún motivo llegan a carecer de los principios indispensables de higiene. ${ }^{5}$

Estas infecciones, además de afectar la salud del personal y de los pacientes, incrementan los costos directos e indirectos, derivados del aumento de días estancia hospitalarias, así como incapacidades prolongadas y en el ámbito laboral la pérdida de horas hombre. Esta situación agrava el desequilibrio existente entre la asignación de recursos para atención primaria y secundaria, al desviar escasos fondos hacia el tratamiento de afecciones potencialmente prevenibles. ${ }^{6}$

En este contexto, para la prevención de las IN, es necesario que el estudiante de enfermería conozca qué son y cómo se trasmiten, para así romper la vía de contagio de manera consciente durante su práctica profesional. Básicamente, dicho conocimiento se compone de higiene, limpieza, desinfección, esterilización, precauciones estándar y aislamiento por mecanismo de transmisión que debe asegurar el espacio del trabajo para el cuidado de la salud. ${ }^{7}$

Según un estudio llevado a cabo en 2015, en la Universidad de Extremadura, relacionado con las medidas de prevención de la transmisión de infecciones nosocomiales y bioseguridad: percepción de los estudiantes. Los resultados obtenidos ponen de manifiesto que el alumnado posee un elevado conocimiento sobre medidas de prevención de la transmisión de infecciones asociadas 
a la actividad sanitaria y prevención, aunque mejorable en algunos aspectos relativos a la higiene de manos, uso de guantes y eliminación de materiales punzocortantes. ${ }^{8}$

Acevedo, et. al. ${ }^{9}$, comparan los conocimientos de dos universidades, cuando se describe la situación que guarda el lavado de manos; en tres momentos evaluados por este estudio, el primer momento se refiere al lavado antes de iniciar algún procedimiento, obteniendo como resultado que el $20 \%$ no lo lleva a cabo. En el segundo momento (contacto con el paciente) el $40 \%$ no lo hace; en relación al tercer momento (término de la atención del paciente) el $40 \%$ de los estudiantes dejan de realizar el procedimiento.

Actualmente, se reconoce la necesidad de establecer mecanismos permanentes de vigilancia epidemiológica que permitan el manejo ágil y eficiente de la información necesaria para la prevención y el control de las infecciones nosocomiales por parte de los estudiantes de enfermería como miembros activos del equipo interdisciplinario de salud, ya que son pieza clave en la atención de pacientes y ejercen liderazgo en la promoción, conservación y restablecimiento de la salud en el medio hospitalario. Por lo que el objetivo de la investigación es Indagar el conocimiento sobre las medidas de prevención en estudiantes de primer a octavo semestre de la Licenciatura de Enfermería de la Universidad Autónoma de Querétaro.

\section{| | | | | | | | | | | | | | | | | | | | | | | | | | | | | | | | | | | | | | | | | | | | | | | | | | | | | | | | | | | | | | | | | | | | | | | | | | | | | | | | | | | | | | | | | | | | | | | | | | | | | | | | |}

\section{Material y métodos}

Se diseñó un estudio trasversal descriptivo, participaron 150 estudiantes de primer a octavo semestre de la Licenciatura de Enfermería de la Universidad Autónoma de Querétaro, seleccionados de forma aleatoria. Para la recolección de datos sociodemográficos se elaboró una cédula que incluye edad, sexo y estado civil y se aplicó el cuestionario "Nivel de conocimiento sobre infecciones nosocomiales y su prevención" de Anaya Flores (2009). El cuestionario consta de dos apartados: co- nocimiento de infecciones nosocomiales y conocimiento de precauciones estándar/ mecanismos de transmisión, con base en las medidas preventivas de infecciones nosocomiales de acuerdo con la Norma Oficial Mexicana NOM-026-SSA2-1998, para la vigilancia epidemiológica, prevención y control de las infecciones nosocomiales. Se realizó análisis estadístico descriptivo para la presentación de la información. Del mismo modo, se consideraron aspectos éticos para garantizar la autonomía y confidencialidad de los participantes.

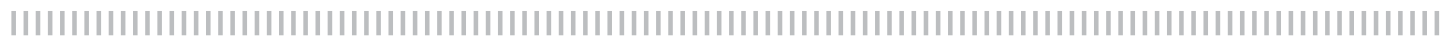

\section{Resultados}

En la tabla 1 se muestran los datos de las variables sociodemográficas de los 150 alumnos participantes, el $88.7 \%$ son menores de 24 años, el $84.7 \%$ son mujeres, y el $85.3 \%$ son solteros. 


\section{Tabla I}

\section{Datos socio demográficos de participantes en el estudio}

\begin{tabular}{|llrr|}
\hline Variable & & Frecuencia & Porcentaje \\
\hline Edad & Menor de 24 años & 133 & 88.7 \\
\hline & Mayor de 25 años & 17 & 11.3 \\
\hline Sexo & Hombre & 23 & 15.3 \\
\hline & Mujer & 127 & 84.7 \\
\hline Condición de unión & Soltero & 128 & 85.3 \\
\hline & Con pareja & 22 & 14.7 \\
\hline Fuente: Cédula de recolección de datos & & $n=150$ \\
\hline
\end{tabular}

En relación con los aspectos relacionados con la prevención de IN, se observa que el total de alumnos considera importante el lavado de manos antes de cualquier procedimiento con el paciente, el método más conocido para evitar la transmisión de microorganismos de una persona a otra, es el lavado de manos con agua y jabón sin embargo, $82.7 \%$ mencionan que, al usar guantes, ya no lo realizan (tabla 2 ).

\section{Tabla 2}

\section{Respuesta de los estudiantes sobre el lavado de manos.}

\begin{tabular}{|llrr|}
\hline Variable & & Frecuencia & \% \\
\hline El lavado de manos al realizar procedimientos invasivos & $\mathrm{Si}$ & 150 & 100 \\
\hline & $\mathrm{No}$ & 0 & 0 \\
\hline El lavado de manos antes y después de lavar una herida & $\mathrm{Si}$ & 150 & 100 \\
\hline & $\mathrm{No}$ & 0 & 0 \\
\hline El lavado de manos no es necesario cuando se usan guantes & $\mathrm{Si}$ & 26 & 17.3 \\
\hline & $\mathrm{No}$ & 124 & 82.7 \\
\hline El lavado de manos al realizar diferentes procedimientos al mismo paciente & $\mathrm{Si}$ & 142 & 94.7 \\
\hline & $\mathrm{No}$ & 8 & 5.3 \\
\hline El lavado de manos previene la trasmisión & $\mathrm{Si}$ & 147 & 98 \\
\hline & $\mathrm{No}$ & 3 & 2 \\
\hline Fuente: Anaya-Flores, 2009 & & $n=150$
\end{tabular}

Por otra parte, los alumnos de los primeros semestres que aún no ha tenido la aprehensión del conocimiento teóricopráctico referente al lavado de manos, como se espera que lo tengan los alumnos de los últimos semestres, respondieron de manera correcta estas preguntas, sin embargo, cuando se les cuestiona sobre el lavado de manos antes y después de usar guantes, contestan de manera errónea (tabla 3).
Referente al conocimiento sobre infecciones nosocomiales y precauciones estándar (tabla 4), los estudiantes manifiestan conocer estos conceptos. Sin embargo, el $16 \%$ mencionó haber padecido una infección nosocomial al llevar a cabo la práctica clínica, haciendo referencia a gastroenteritis, faringoamigdalitis y conjuntivitis como las infecciones adquiridas en sus prácticas hospitalarias. 


\section{Tabla 3}

Conocimiento sobre lavado de manos según semestre.

\begin{tabular}{|c|c|c|c|c|c|}
\hline & \multicolumn{5}{|c|}{ Semestre } \\
\hline & & \multicolumn{2}{|l|}{$1-4$} & \multicolumn{2}{|c|}{$5-8$} \\
\hline & & Frecuencia & Porcentaje & frecuencia & porcentaje \\
\hline \multirow[t]{2}{*}{ El lavado de manos al realizar procedimientos invasivos } & No & 0 & 0 & 0 & 0 \\
\hline & $\mathrm{Si}$ & 76 & 100 & 74 & 100 \\
\hline \multirow[t]{2}{*}{ El lavado de manos antes y después de lavar una herida } & No & 0 & 0 & 0 & 0 \\
\hline & $\mathrm{Si}$ & 76 & 100 & 74 & 100 \\
\hline \multirow[t]{2}{*}{$\begin{array}{l}\text { El lavado de manos no es necesario cuando se } \\
\text { usan guantes }\end{array}$} & No & 64 & 84.2 & 60 & 81.1 \\
\hline & $\mathrm{Si}$ & 12 & 15.8 & 14 & 18.9 \\
\hline $\begin{array}{l}\text { El lavado de manos al realizar diferentes } \\
\text { procedimientos al mismo paciente }\end{array}$ & $\begin{array}{l}\mathrm{No} \\
\mathrm{Si}\end{array}$ & $\begin{array}{c}5 \\
71 \\
\end{array}$ & $\begin{array}{c}6.6 \\
93.4 \\
\end{array}$ & $\begin{array}{c}3 \\
71 \\
\end{array}$ & $\begin{array}{r}4.1 \\
95.9 \\
\end{array}$ \\
\hline $\begin{array}{l}\text { El lavado de manos previene la transición de } \\
\text { infecciones de paciente a paciente }\end{array}$ & $\begin{array}{l}\mathrm{No} \\
\mathrm{Si}\end{array}$ & $\begin{array}{c}1 \\
75 \\
\end{array}$ & $\begin{array}{c}1.3 \\
98.7 \\
\end{array}$ & $\begin{array}{c}2 \\
72 \\
\end{array}$ & $\begin{array}{r}2.7 \\
97.3 \\
\end{array}$ \\
\hline Fuente: Anaya-Flores, 2009 & & & & & $n=150$ \\
\hline
\end{tabular}

\section{Tabla 4}

\section{Conocimiento sobre infecciones nosocomiales y precauciones estándar.}

\begin{tabular}{|lccc|}
\hline Variable & & Frecuencia & $\%$ \\
\hline Riesgo de adquirir una infección nosocomial & $\mathrm{Si}$ & 147 & 98.0 \\
\hline & $\mathrm{No}$ & 3 & 2.0 \\
\hline Adquisición de alguna infección nosocomial & $\mathrm{Si}$ & 24 & 16.0 \\
\hline & $\mathrm{No}$ & 126 & 84.0 \\
\hline Conozco las precauciones estándar para prevenir infecciones nosocomiales & $\mathrm{Si}$ & 127 & 84.7 \\
\hline & $\mathrm{No}$ & 23 & 15.3 \\
\hline Las precauciones estándar, son necesarias para prevenir infecciones nosocomiales & $\mathrm{Si}$ & 149 & 99.3 \\
\hline & $\mathrm{No}$ & 1 & 0.7 \\
\hline Conozco ejemplos de infecciones nosocomiales & $\mathrm{Si}$ & 124 & 82.7 \\
\hline & $\mathrm{No}$ & 26 & 17.3 \\
\hline Fuente: Anaya-Flores, 2009 & & & $n=150$
\end{tabular}

Al realizar la comparativa (tabla 5), se observa que los alumnos de primero y octavo conocen las precauciones estándar, pero aun así, el $15.8 \%$ de los primeros se- mestres también refieren haber padecido durante el periodo de prácticas alguna de las patologías descritas en la tabla 4. 


\section{Tabla 5}

Conocimiento por semestre de infecciones nosocomiales y precauciones estándar.

\begin{tabular}{|llcccc|}
\hline \multicolumn{1}{|c}{ Semestre } \\
\hline \multicolumn{1}{|c}{ Variable1-4 } & \multicolumn{5}{c}{$5-8$} \\
\hline & & Frecuencia & Porcentaje & frecuencia & porcentaje \\
\hline Riesgo de adquirir una infección nosocomial & $\mathrm{No}$ & 2 & 2.6 & 1 & 1.4 \\
\hline & $\mathrm{Si}$ & 74 & 97.4 & 73 & 98.6 \\
\hline Adquisición de alguna infección nosocomial & $\mathrm{No}$ & 64 & 84.2 & 62 & 83.8 \\
\hline & $\mathrm{Si}$ & 12 & 15.8 & 12 & 16.2 \\
\hline $\begin{array}{l}\text { Conozco las precauciones estándar para prevenir } \\
\text { infecciones nosocomiales }\end{array}$ & $\mathrm{No}$ & 6 & 7.9 & 6 & 8.1 \\
\hline Las precauciones estándar, son necesarias para & $\mathrm{Si}$ & 70 & 92.1 & 68 & 91.9 \\
prevenir infecciones nosocomiales & $\mathrm{No}$ & 0 & 0 & 1 & 1.4 \\
\hline Conozco ejemplos de infecciones nosocomiales & $\mathrm{Si}$ & 76 & 100 & 73 & 98.6 \\
\hline & $\mathrm{No}$ & 6 & 7.9 & 20 & 27 \\
\hline
\end{tabular}

Fuente: Anaya-Flores, 2009 $n=150$

| | | | | | | | | | | | | | | | | | | | | | | | | | | | | | | | | | | | | | | | | | | | | | | | | | | | | | | | | | | | | | | | | | | | | | | | | | | | | | | | | | | | | | | | | | | | | | | | | | | | |

\section{Discusión}

El conocimiento para la prevención de infecciones coadyuva en la reducción de la morbilidad y mortalidad asociada a infecciones mediante estrategias básicas, como la práctica de las precauciones estándar en los pacientes hospitalizados independientemente de su diagnóstico según lo mencionar Anaya Flores (2009), situación que se reporta en nuestro estudio los estudiantes refieren en su totalidad, conocer las precauciones estándar, sin embargo, en la práctica modifican su actuar, al buscar una protección personal desde el momento en que aducen que si se utilizan guantes ya no es "necesario" el lavado de manos $(81 \%)$. Situación que difiere de lo reportado por Acevedo, et.al. ${ }^{9}$ quien menciona que el $20 \%$ no lo realiza.

Los guantes son una barrera física que protege, tanto a los trabajadores de los servicios sanitarios, así como a los usuarios, por lo que es indispensable que los guantes contaminados sigan el procedimiento establecido para Recolección de productos biológicos infecciosos (RPBI), y el personal de salud deberá concluir con el lavado de manos ${ }^{10,11}$. Es importante mencionar que los estudiantes participantes conocen las medidas estándar (91.9\%); pero eso no garantiza la aplicación del procedimiento.

En relación con la infección nosocomial, definida como toda aquella adquirida durante la hospitalización y que se desarrolla en un paciente después de 48-72 horas de atención hospitalaria, ${ }^{11}$ el $16 \%$ de los estudiantes refieren haber adquirido una infección en la práctica, lo anterior nos conduce a pensar que si bien los estudiantes demuestran conocer los conceptos, ello no quiere decir que los apliquen de manera consciente e intencionada, es decir, es muy probable que no estén concretando saberes de tipo procedimental y actitudinal.

Si se considera que el personal de enfermería es quien permanece más tiempo al lado del enfermo, proporcionándole un cuidado permanente para esto se requieren altos conocimientos, habilidades y actitudes; por ello es de gran importancia resaltar el rol que cumple el profesional de enfermería al educar y cuidar al paciente, la familia y los colectivos, para coadyuvar en la modificación de los procesos de riesgo y en las estrategias de intervención desde la promoción de la salud, la preven- 
ción de la enfermedad y la educación a la comunidad. ${ }^{12}$

Por lo que es de suma importancia la supervisión y adiestramiento en servicio durante las prácticas clínicas por parte de los docentes, para identificar áreas de oportunidad en relación con actividades relacionadas con la prevención de IN.

Asimismo, enfatizar las acciones para el cuidado que realizan los estudiantes y personal de enfermería con los pacientes en los que se conoce o se sospecha de la existencia de un tipo específico de enfermedad infecciosa. Por otra parte, el control de equipos estériles, el uso adecuado de desinfectantes, la limpieza de aéreas físicas, son necesarias para evitar infecciones cruzadas de paciente a paciente.

Este estudio permitió identificar el grado de conocimiento por nivel académico de los estudiantes de la Licenciatura en Enfermería en relación con las medidas de prevención en IN. De acuerdo a los resultados arrojados el nivel de conocimientos es regular, considerando que es de vital importancia, en el ejercicio del área de la salud, el proceso de enseñanza aprendizaje holístico (saber conocer, saber hacer y saber ser) para reforzar el conocimiento sobre el manejo de las normas de bioseguridad, ya que esto garantiza conservar su integridad física y mental "al hacer lo que debe hacer y cómo debe ser", sin poner en riesgo su vida ni la de sus pacientes.

Para conocer los factores que conducen a los estudiantes a incumplir estas normas, se debe explorar y revisar bajo qué condiciones realizan sus prácticas clínicas, como un elemento clave en la formación de los recursos humanos, con los conocimientos habilidades y destrezas necesarias para dar cumplimiento a las medidas estándar y la prevención de IN.

\section{Conclusiones}

Para conocer los factores que conducen a los estudiantes al incumplimiento de estas normas, es necesario revisar las condiciones en que se realiza la práctica clínica, para garantizar la calidad en la formación de recursos humanos para la salud.

\section{Bibliografía}

1. Norma Oficial Mexicana de Emergencia NOM-EM002-SSA2-2003, Para la Vigilancia Epidemiológica, Prevención y Control de las Infecciones Nosocomiales.

2. Nodarse Hernández, R. Visión actualizada de las infecciones intrahospitalarias. Revista Cubana de Medicina Militar, 2002; 31(3), 201-208.

3. Lebeque Pérez, Y., Morris Quevedo, H. J., \& Calás Viamonte, N. Infecciones nosocomiales: incidencia de la Pseudomonas aeruginosa. Revista Cubana de medicina, 2006; 45(1), 0-0 4

4. Cardo D, Dennehy PH, Halverson P, Fishman N, Kohn $M$, Murphy $C L$ Whitley $\amalg$, Moving toward elimination of healthcare-associated infections: A call to action. Am J Infect Control 2010; 38:671-5

5. Brenner P, Bugedo G, Calleja D, Del Valle G, Fica A, \& Gómez, O. M. E. Prevención de infecciones asociadas a catéteres vasculares centrales. Rev Chil Infect, 2008; 20(1), 51-69

6. NOM-045-SSA2-2005, Para la vigilancia epidemiológica, prevención y control de las infecciones nosocomiales.

7. Brenner, P. Op. Cit.

8. Castillo, J. Medidas de transmisión de infecciones nosocomiales de bioseguridad: percepción de los estudiantes del grado de enfermería, Tesis doctoral, España: Universidad de Extremadura; 2015.

9. Acevedo, K.P.; Coha, K.L.; Ortiz, A.M. Evaluación del conocimiento y aplicación de las normas de bioseguridad en estudiantes de enfermería del último año en el servicio de medicina interna de dos IES Cartagena de Indias. Tesis de grado, Licenciatura, Cartagena de Indias, Colombia: Corporación Universitaria Rafael Núñez; 2013. 
10. Anaya Flores V y Valencia Martínez, J. Infecciones nosocomiales en un hospital de alta especialidad. Factores asociados a mortalidad. Rev. Med IMSS, 2007; 43(5), 381-391.

11. Dirección de servicios sanitarios. Coordinadora enfermería. Protocolo de Lavado de manos y uso correcto de guantes en Atención Primaria de Asturias, $1^{\text {a }}$ edición 15-07-2009. Disponible desde: https://www. asturias.es/Astursalud/Ficheros/AS_SESPA/AS_Ges- tion\% 20Clinica/AS Seguridad\% 20Paciente/Protocolo\% 20Lavado\% 20M̄anos\% 20AP.pdf

12. Bran de Casares A, Díaz de Rivera C, Martínez D, Rubida Girón F, García Arévalo G, De Jesús Mochica L. Lineamientos Técnicos en la Prevención y Control de las Infecciones Nosocomiales [Internet]. 2006 marzo [citado 2015 enero 15]; pp67. Disponible desde: http://asp.salud.gob.sv/regulacion/pdf/manual/Manual nosocomiales.pdf 